of oculomotor and trochlear nerve involvement have also been reported. ${ }^{3-6}$ With intracranial hypertension, changes in seventh nerve function are exceptional. Peripheral facial nerve palsy was observed in five individuals suffering from benign intracranial hypertension. ${ }^{78}$ In one additional patient facial myokymia has been observed, thus showing that, with intracranial hypertension, facial nerve involvement can result in axonal hyperactivity.

No clear explanation was offered as to the pathophysiology of the facial nerve involvement in intracranial hypertension, although it was felt that this dysfunction represented a non-specific pressure related phenomenon. In our patient, it is conceivable that the displacement of intracranial nervous structures related to the Arnold-
Chiari type I malformation played a role in the mechanism of this peculiar eyelid phenomenon, by allowing the seventh cranial nerve to be affected by elevation in intracranial pressure.

1 Feindel W, Penfield W, McNaughton F. The tentorial nerves and localisation of intracranial pain in man. Neurology 1960 10: $555-63$

2 Johnston I, Paterson A. Benign intracranial hypertension. Brain 1974; 97: 289-300.

3 Kiwak KJ, Levine SE. Benign intracranial hypertension and facial diplegia. Arch Neurol Vol 1984; 41: 787-8.

4 Walsh FB, Hoyt WF. In: Clinical neuro-ophthalmology 3rd ed. Baltimor: Williams \& Wilkins, 1969. $198,498,593,1932-6$.

Baltimore. Whiams \& Wilkins, 1969. 198, 498, 593, 1932-6.

McCammon A, Kaufman HH, Sears SE. Transient oculomoto paralysis in pseudotumor cerebri. Neurology $1981 ; 31: 182-4$. Halpern JI, Gordon WH. Trochlear nerve palsy as a false localizing sign. Ann Ophthalmol 1981; 13: 53-6.

7 Snyder DA, Frenkel M. An unusual presentation of pseudotumor cerebri. Ann Ophthalmol 1979; 11: 1823-7.

8 Chutorian AB, Gold AP, Braun CW. Benign intracranial hypertension and Bell's palsy. N Engl f Med 1977; 26: 1214

\title{
Fuchs' heterochromic uveitis associated with retinitis pigmentosa in a father and son
}

\author{
L Ingeborgh van den Born, Mary J van Schooneveld, Paulus T V M de Jong, \\ Elisabeth $M$ Bleeker-Wagemakers
}

Fuchs' heterochromic uveitis (FHU) in patients with retinitis pigmentosa $(\mathrm{RP})$ is a rare entity. Only five cases with this combination have been reported in literature. ' In four of these cases FHU was associated with simplex RP. In one case the retinal degeneration was demonstrated to be of autosomal recessive inheritance.'

We report on the coexistence of autosomal dominant RP and unilateral FHU in a father and son. RP as well as FHU are relatively infrequent diseases. The prevalence of RP is 25 in 100000 , while the dominant form accounts for less than $25 \%$. $^{2} \mathrm{FHU}$ is even more exceptional, since its prevalence is estimated at 1.8 in $100000 .^{3}$

Department of

Ophthalmogenetics, The Netherlands Ophthalmic Research Institute, Amsterdam, The

Netherlands

L I van den Born

$M J$ van Schooneveld

E M Bleeker-Wagemakers

Institute of

Ophthalmology, Erasmus

University, Rotterdam,

The Netherlands

P T V M de Jong

Correspondence to:

$L$ Ingeborgh van den Born,

Department of

Ophthalmogenetics, The

Netherlands Ophthalmic

Research Institute, Postbox

12141,1100 AC Amsterdam,

The Netherlands.

Accepted for publication

1 February 1994

\section{Case reports}

Case II-3 (Fig 1) was a healthy 56-year-old man with a history of night blindness from early childhood. At 29 years of age visual acuities were 0.25 in the right eye and hand movements in the left eye. Slit-lamp examination of the left eye revealed keratic precipitates, iris atrophy with a darker coloured iris than in the right eye (inversed heterochromia), and a dense cataract. Posterior synechiae were absent. The anterior segment of the right eye was unremarkable. On funduscopy he had pale optic discs, attenuated vessels, and peripheral bone corpuscle-shaped pigmentations in both eyes. The right visual field was constricted to 30 degrees central vision. There were no symptoms of redness, pain, or photophobia. Case II-3 fulfilled all criteria of unilateral FHU and bilateral RP. After this initial examination an intracapsular lens extrac- tion was performed. Throughout the years the keratic precipitates have always been present. Intraocular pressures remained normal. On the most recent examination visual acuity was $0 \cdot 125$ in the right eye and light perception in the left aphakic eye. A small anterior and posterior subcapsular cataract was present in the right lens. Both vitreous cavities contained cells.

The proband's healthy 33-year-old son, case III-5, was noticed to be night blind from the age of 3 . When he was 14 years of age the diagnosis RP was established. Five years later keratic precipitates were noticed in the left eye, together

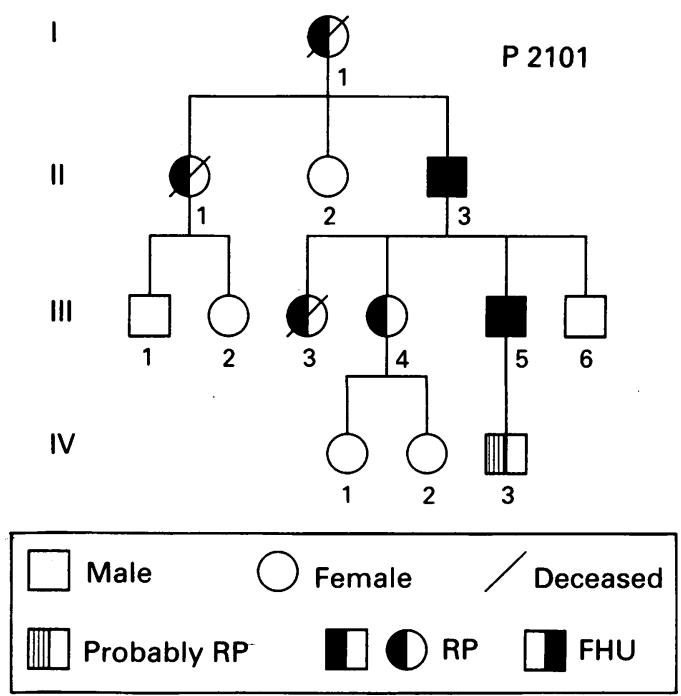

Figure 1 The family pedigree. 
with cells and a slight flare in the anterior chamber. A systemic cause for the unilateral uveitis could not be detected. Another 6 years later diffuse atrophy of the iris pigment layer with diaphany became obvious and FHU was diagnosed. On the most recent examination visual acuity was 0.4 in the right eye and 0.3 in the left eye. Like his father he had keratic precipitates and inversed heterochromia. There were no posterior synechiae. The posterior subcapsular cataract in the left eye was larger than in the right one. Both vitreous cavities contained cells. Fundus abnormalities were symmetrical and included pale optic discs, attenuation of the vessels, and round and bone corpuscle-shaped pigmentations in the periphery. Intraocular pressures were normal. Visual fields were constricted to 10 degrees in both eyes.

Case III-4, 34 years of age, had exactly the same fundus abnormalities as her brother, but no symptoms indicating FHU. Cases I-1, II-1, and III-3, were all known as RP patients. From their medical files no features suggesting FHU were detected. The 3-year-old son (case IV-3) of case III-5 was noticed to be night blind. On funduscopy the arterioles appeared attenuated with mild pigmentary changes in the periphery. No electroretinogram had been performed yet. Cases III-1, III-2, and III-6 who were all over the age of 30 were not affected by either RP or FHU. Cases IV-1 and IV-2 were younger than 10 years and had no abnormalities at the time of examination.

\section{Comment}

To our knowledge this is the first report on the coexistence of RP and unilateral FHU in two family members. The dominant RP in the family described was of an early onset form with night blindness from childhood and macular involvement before 20 years of age. Besides their RP, the father and his son fulfilled all criteria of FHU as put forward by Franceschetti. ${ }^{4}$ The posterior subcapsular cataracts, although a known finding in $\mathrm{RP},{ }^{2}$ were more prominent in the eyes with the FHU. The occurrence of vitreous cells was symmetrical in the right and left eye of both persons, but is common in RP patients ${ }^{2}$ and thus could not be used as a criterion for FHU.

Five years after the identification of $\mathrm{RP}$ in case III-5, the first symptoms for FHU were noted.
This might suggest that the FHU was secondary to the retinal degeneration, but would not explain why the FHU was unilateral. Moreover, only five previous cases of RP associated with FHU have been reported,' which gives the impression of coincidental occurrence of both diseases. Considering the prevalences of RP and FHU, however, seven cases with coexistence of $\mathrm{RP}$ and FHU is already one third of the number of cases to be expected worldwide. Therefore, a causal relation between the two diseases cannot be excluded entirely.

The father-to-son transmission of the FHU suggests, just like the RP, an autosomal dominant trait. Jones and $\operatorname{Read}^{5}$ recently stated that Mendelian inheritance of FHU was impossible, based on the discordance in one pair of monozygotic twins. However, rejection of the Mendelian trait based on one pair of monozygotic twins is not very convincing, since nonpenetrance is difficult to exclude and the opposite, identical twins with FHU, have also been described. ${ }^{6}$ Moreover Jones and Read ${ }^{5}$ examined only accessible relatives with a history of heterochromia or inflammatory eye disease, but since FHU patients may be asymptomatic and heterochromia can be subtle or absent, ${ }^{+}$ familial cases might have been missed. Although familial occurrence of FHU is low, our two cases support the possibility of a genetic predisposition. This might not necessarily be a Mendelian trait. HLA subtypes which are strongly linked with other types of uveitis, do not form a predisposition for $\mathrm{FHU}^{+}$Whether or not a genetic predisposition for FHU can come to expression by events like a retinal degeneration, is speculative. A hereditary factor closely linked to RP did not seem likely in our pedigree, since the other RP cases had no signs of FHU, and therefore we presume that both traits segregate independently.

1 Vuorre I, Saari M, Tiilikainen A, Rasanen O. Fuchs' heterochromic cyclitis associated with retinitis pigmentosa: a family study. Canad $\mathcal{F}$ Ophthalmol 1979; 14: 10-6.

2 Heckenlively JR. Retinitis pigmentosa. Philadelphia: Lippincott, 1988: 21, 75, 77 .

3 Vadot E, Barth E, Billet P. Epidemiology of uveitis - preliminary results of a prospective study in Savoy. In: Saar KM, ed. Uveitis update. Amsterdam: Elsevier, 1984: 13.6.

4 Jones NP. Fuchs' heterochromic uveitis: an update. Survey 1993; 37: 253-72.

5 Jones NP, Read AP. Is there a genetic basis for Fuchs' heterochromic uveitis? Discordance in monozygotic twins. Br f Ophthalmol 1992; 76: 22-4.

6 Makley TA. Heterochromic cyclitis in identical twins. Am $\mathcal{J}$ Ophthalmol 1956; 41: 768-72. 